\title{
ABKÜRZUNGEN UND SIGEL
}

AA. Archăologischer Anzeiger

AAel. Archaeologia Aeliana

AAJ. Annual of the Department of Antiquities of Jordan

AASOR. Annual of the American Schools of Oriental Research in Jerusalem

AASyr. Les Annales Archéologiques de Syrie

AbhBerlin Abhandlungen der Deutschen (PreuBischen) Akademie der Wissenschaften zu Berlin. Phil.-hist. Klasse

AbhHeidelberg Abhandlungen der Heidelberger Akademie der Wissenschaften. Phil.-hist. Klasse

AbhLeipzig Abhandlungen der (Königlich) Sächsischen Gesellschaft der Wissenschaften. Phil.hist. Klasse

AbhMainz Akademie der Wissenschaften und der Literatur in Mainz. Abhandlungen der geistesund sozialwissenschaftl. Klasse

AbhMünchen Abhandlungen der (Königlich) Bayerischen Akademie der Wissenschaften. Hist. Klasse

ABr. Griechische und Römische Porträts (,ArndtBruckmann")

ACambr. Archaeologia Cambrensis

ActaArch. Acta Archaeologica (Kopenhagen)

ActaIRN. Institutum Romanum Norvegiae. Acta ad Archaeologiam et Artium Historiam pertinentia

ActMuz. Activitatea Muzeelor, Cluj

AD. Antike Denkmäler

ADIK. Abhandlungen des Deutschen Archäologischen Instituts Abteilung Kairo

AeR. Atene e Roma

AEsp. Archivo Español de Arqueologia

A JA. American Journal of Archaeology

AJPh. American Journal of Philology

AIPhOr. Annuaire de l'Institut de Philologie et d'Histoire Orientales et Slaves

Akten IOK. Akten des Internationalen Orientalisten-Kongresses, München

AM. Mitteilungen des Deutschen Archăologischen Instituts. Athenische Abteilung

Amelung, Vat. Kat. W. Amelung, Die Sculpturen des Vaticanischen Museums
AnatSt. Anatolian Studies

ANL. Archaeological News Letter

AnnFABelg. Annales, Fédération Archéologique et Historique de Belgique

AnnIstNum. Istituto italiano di Numismatica. Annali

AnnPisa Annali della Reale Scuola Normale Superiore di Pisa. Sezione di Lettere

AntCl. L'Antiquité Classique

AntJ. The Antiquaries Journal

AntK. Antike Kunst

AnzAW. Anzeiger für die Altertumswissenschaft

AnzWien Anzeiger der Akademie der Wissenschaften, Wien. Phil.-hist. Klasse

AO. Der Alte Orient

AOF. Archiv für Orientforschung

AOr. Archiv Orientálni

APort. O Arqueólogo Portuguès

ArchCl. Archeologia Classica

ArchErt. Archaeologiai Ertesitö

Arch J. The Archaeological Journal

ArchStorCalabria Archivio Storico per la Calabria e la Lucania

ArchStorSicilia Archivio Storico per la Sicilia Orientale

ArtB. The Art Bulletin

ASAntEg. Annales du Service des Antiquités de l'Égypte

ASAtene Annuario della Scuola Archeologica di Atene

AttiCIAC. Atti del Congresso Internazionale di Archeologia Classica

AttiMGrecia Atti e Memorie della Società Magna Grecia

AttiSocTiburtina Atti e Memorie della Società Tiburtina di Storia e d'Arte

AuA. Antike und Abendland

AvP. Altertümer von Pergamon

AZ. Archäologische Zeitung

BA. Bulletin Archéologique du Comité des Travaux Historiques et Scientifiques

BAcBelg. Académie Royale de Belgique. Bulletin de la Classe des Lettres 
BadFB. Badische Fundberichte

BAntBeschav. Bulletin van de Vereeniging tot Bevordering der Kennis van de Antieke Beschaving te 's Gravenhage

BAntFr. Bulletin ae la Société Nationale des Antiquaires de France

BArchAlex. Bulletin de la Société Archéologique d'Alexandrie

BASOR. Bulletin of the American Schools of Oriental Research in Jerusalem

BayVBll. Bayerische Vorgeschichtsblätter

BBudé Bulletin de l'Association Guillaume Budé

BBudéSCr. Bulletin de l'Association Guillaume Budé. Supplément Critique

$\mathrm{BCH}$. Bulletin de Correspondance Hellénique

Bd'A. Bollettino d'Arte

Beazley, ABV. J. D. Beazley, Attic Black-Figure Vase-Painters

Beazley, ARV. J. D. Beazley, Attic Red-Figure Vase-Painters

Benndorf-Schöne O. Benndorf - R. Schöne, Die antiken Bildwerke des Lateranensischen Museums

BerlMus. Berliner Museen. Berichte aus den Ehem. PreuB. Kunstsammlungen

BerRGK. Deutsches Archäolog. Institut. Bericht der Römisch-Germanischen Kommission

BerROB. Berichten van de Rijksdienst voor Oudheidkundig Bodemonderzoek

BerSaarland Bericht der Staatlichen Denkmalpflege im Saarland

BJb. Bonner Jahrbücher

BiblA. The Biblical Archaeologist

BIBulg. Bulletin de l'Institut Archéologique Bulgare

BICS. Bulletin of the Institute of Classical Studies, University of London

BIE. Bulletin de l'Institut Égyptien

BIFAO. Bulletin de l'Institut Français d'Archéologie Orientale

BiOr. Bibliotheca Orientalis

BMC. A Catalogue of the Greek Coins in the British Museum

BMetrMus. Bulletin of the Metropolitan Museum of Art

BMusArt Bulletin des Musées Royaux d'Art et d'Histoire

BMusBeyr. Bulletin du Musée de Beyrouth

BMusHongr. Bulletin du Musée Hongrois des Beaux-Arts

BollICR. Bollettino dell' Istituto Centrale del Restauro

BollMC. Bollettino dei Musei Comunali di Roma
BPI. Bullettino di Paletnologia Italiana

BrBr. Brunn-Bruckmann's Denkmäler griechischer und römischer Skulptur

BrMQu. The British Museum Quarterly

BSA. Annual of the British School at Athens

BSR. Papers of the British School at Rome

BullCom. Bullettino della Commissione Archeologica Comunale di Roma

BullMCR. Bullettino dei Musei Comunali Romani

BVarna Bulletin de la Société Archéologique à Varna

BWPr. Winckelmannsprogramm der Archäologischen Gesellschaft zu Berlin

ByzZ. Byzantinische Zeitschrift

CAlsArch. Cahiers Alsaciens d'Archéologie, d'Art et d'Histoire

CArch. Cahiers Archéologiques

CHistMond. Cahiers d'Histoire Mondiale

ChrEg. Chronique d'Égypte

CIG. Corpus Inscriptionum Graecarum

CIL. Corpus Inscriptionum Latinarum

C1B. The Classical Bulletin

ClJ. The Classical Journal

ClPh. Classical Philology

ClQu. The Classical Quarterly

ClRev. The Classical Review

ClW. The Classical Weekly

CorsiRavenna Corsi di Cultura sull'Arte ravennate e bizantina

CRAcInscr. Comptes-Rendus des Séances. Académie des Inscriptions et Belles-Lettres

Crd'A. La Critica d'Arte

CVA. Corpus Vasorum Antiquorum

Daremberg-Saglio Dictionnaire des Antiquités Grecques et Romaines ... sous la Direction de Ch. Daremberg et Edm. Saglio

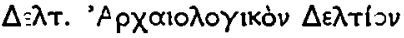

DLZ. Deutsche Literaturzeitung

EA. Photographische Einzelaufnahmen antiker Skulpturen ("Arndt-Amelung“)

EAZ. Ethnographisch-Archäologische Zeitschrift

Ebert, RV. Reallexikon der Vorgeschichte, hrsg. von M. Ebert

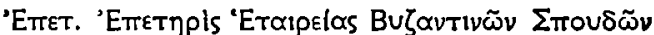

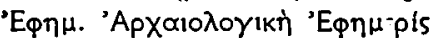

EtAC. Études d'Archéologie Classique

EtCl. Les Études Classiques

EUA. Enciclopedia Universale dell'Arte 
FA. Fasti Archaeologici

FBHessen Fundberichte aus Hessen

FBSchwaben Fundberichte aus Schwaben

FdD. Fouilles de Delphes

FolArch. Folia Archaeologica

FR. A. Furtwängler - K. Reichhold, Griechische Vasenmalerei

FuB. Forschungen und Berichte der Staatl. Museen zu Berlin

FuF. Forschungen und Fortschritte

GaR. Greece and Rome

GazBA. Gazette des Beaux-Arts

GGA. Göttingische Gelehrte Anzeigen

GlMSar. Glasnik Zemalskog Muzeja u Sarajevü

GodPlovdiv Godišnik na Narodnija Archeolog. Musei Plovdiv

Guida Ruesch A. Ruesch, Guida Illustrata del Museo Nazionale di Napoli

HallWPr. Hallisches Winckelmannsprogramm

HarvSt. Harvard Studies in Classical Philology

HAW. Handbuch der (klassischen) Altertumswissenschaft. Begründet von Iwan Müller, hrsg. von W. Otto

$\mathrm{HBr}$. P. Herrmann, Denkmäler der Malerei des Altertums (,Herrmann-Bruckmann“)

HdArch. Handbuch der Archäologie im Rahmen des Handbuchs der Altertumswissenschaft. Hrsg. von W. Otto, fortgeführt von R. Herbig

Helbig ${ }^{3}$ W. Helbig, Führer durch die öffentlichen Sammiungen klassischer Altertümer in Rom. 3. Auflage

HZ. Historische Zeitschrift

JAOS. Journal of the American Oriental Society JAsiat. Journal Asiatique

JbAChr. Jahrbuch für Antike und Christentum

JbBerlin Jahrbuch der Deutschen Akademie der Wissenschaften zu Berlin

JberEOL. Jaarbericht van het Voor-AziatischEgyptische Gezelschap 'Ex Oriente Lux'

JberVind. Jahresbericht, Gesellschaft Pro Vindonissa

JBiblLit. Journal of Biblical Literature

JbKlF. Jahrbuch für Kleinasiatische Forschung JbKSWien Jahrbuch der Kunsthistorischen Sammlungen in Wien

JbLeipzig Jahrbuch, Sächsische Akademie der Wissenschaften zu Leipzig

JbMainz Jahrbuch, Akademie der Wissenschaften und der Literatur, Mainz
JbNum. Jahrbuch für Numismatik und Geldgeschichte

JbÖBG. Jahrbuch der Österreichischen Byzantinischen Gesellschaft

JbSGU. Jahrbuch der Schweizerischen Gesellschaft für Urgeschichte

JbZMusMainz Jahrbuch des Römisch-Germanischen Zentralmuseums, Mainz

JBritArchAss. The Journal of the British Archaeological Association

JdI. Jahrbuch des Deutschen Archäologischen Instituts

JEA. The Journal of Egyptian Archaeology

IEJ. Israel Exploration Journal

IG. Inscriptiones Graecae

JHS. The Journal of Hellenic Studies

ILN. Illustrated London News

JNES. Journal of Near Eastern Studies

JRS. The Journal of Roman Studies

JSav. Journal des Savants

IstForsch. Istanbuler Forschungen

IstMitt. Istanbuler Mitteilungen

JWCI. Journal of the Warburg and Courtauld Institute

LF. Listy filologicke

Lippold, Vat. Kat. G. Lippold, Die Skulpturen des Vaticanischen Museums

MarbJb. Marburger Jahrbuch für Kunstwissenschaft

MarbWPr. Marburger Winckelmann-Programm

Matz-Duhn F. Matz, Antike Bildwerke in Rom mit Ausschluß der größeren Sammlungen. (Weitergef. u. hrsg. von F. von Duhn)

MCArh Materiale si Cercetări Arheologice

MdI. Mitteilungen des Deutschen Archäologischen Instituts

MDIK. Mitteilungen des Deutschen Archäologischen Instituts Abteilung IKairo

MDOG. Mitteilungen der Deutschen Orient-Gesellschaft

Mél. Mélanges d'Archéologie et d'Histoire

MemAccLinc. Atti della Accademia Nazionale dei Lincei. Memorie

MémAcInscr. Mémoires présentés par Divers Savants à l'Académie des Inscriptions et BellesLettres

MemAmAc. Memoirs of the American Academy in Rome

Mém Côte-d'Or Mémoires de la Commission des Antiquités du Dép. de la Côte-d'Or 
MemPontAcc. Atti della Pontificia Accademia Romana di Archeologia. Memorie

MémSocAF. Mémoires de la Société Nationale des Antiquaires de France

MIA. Materialy i Issledovanija po Archeologii SSSR.

MM. Madrider Mitteilungen

MonAnt. Monumenti Antichi pubblicati per Cura della Accademia Nazionale dei Lincei

MonInst. Monumenti Inediti pubblicati dall' Instituto Archeologico

MonPiot Fondation Eugène Piot. Monuments et Mémoires

MüJb. Münchner Jahrbuch der bildenden Kunst

MusHelv. Museum Helveticum

NAHisp. Noticiario Arqueológico Hispánico

NGG. Nachrichten der Akademie der Wissenschaften in Göttingen

NSc. Notizie degli Scavi di Antichità

NumChron. The Numismatic Chronicle and Journal of the Numismatic Society

NumZ. Numismatische Zeitschrift

OJh. Jahreshefte des Österreichischen Archäologischen Instituts in Wien

Olymp. Forschungen Olympische Forschungen. Hrsg. von E. Kunze

Olympia I (-V) Olympia. Die Ergebnisse der von dem Deutschen Reich veranstalteten Ausgrabung... hrsg. von E. Curtius und F. Adler. Band I-V

Olympiabericht I (ff.) I. (ff.) Bericht über die Ausgrabungen in Olympia

OLZ. Orientalistische Literaturzeitung

OpArch. Skrifter utgivna av Svenska Institutet i Rom. Opuscula Archaeologica.

OpAth. Opuscula Atheniensia

OpRom. Opuscula Romana

OudhMeded. Oudheidkundige Mededelingen uit het Rijksmuseum van Oudheden te Leiden

PalExpl Qu. Palestine Exploration Quarterly

Pfuhl, MuZ. E. Pfuhl, Malerei und Zeichnung der Griechen

PhQu. Philological Quarterly

PP. La Parola del Passato

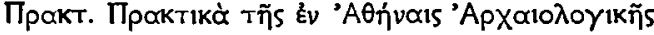
'Etaipelas

ProcACA. Proceedings of the African Classical Associations

ProcCambr. Proceedings of the Cambridge Antiquarian Society
ProcScotland Proceedings of the Society of Antiquaries of Scotland

PrzArch. Przegląd Archeologiczny

PZ. Praehistorische Zeitschrift

RA. Revue Archéologique

RAC. Reallexikon für Antike und Christentum

RACentre Revue Archéologique du Centre

RAComo Rivista Archeologica dell'Antica Provincia e Diocesi di Como

RACrist. Rivista di Archeologia Cristiana

RAE. Revue Archéologique de l'Est et du Centre-Est

RArts Revue des Arts

RAssyr. Revue d'Assyriologie et d'Archéologie orientale

RBArch. Revue Belge d'Archéologie et d'Histoire de l'Art

RBibl. Revue Biblique

RBNum. Revue Belge de Numismatique

RBPhil. Revue Belge de Philologie et d'Histoire

RE. Paulys Realencyclopädie der classischen Altertumswissenschaft. Neue Bearbeitung

REA. Revue des Études Anciennes

REByz. Revue des Études Byzantines

REG. Revue des Études Grecques

REgypt. Revue d'Égyptologie

REL. Revue des Etudes Latines

RendAccNapoli Rendiconti della Reale Accademia di Archeologia, Lettere e Belle Arti di Napoli

RendIstLomb. Istituto Lombardo di Scienze e Lettere. Rendiconti

RendLinc. Atti della Accademia Nazionale dei Lincei. Rendiconti

RendPontAcc. Atti della Pontificia Accademia Romana di Archeologia. Rendiconti

RevABM. Revista de Archivos, Bibliotecas y Museos

RevNum. Revue Numismatique

RevPhil. Revue de Philologie, de Littérature et d'Histoire Anciennes

RGG. Die Religion in Geschichte und Gegenwart

RH. Revue Historique

RhM. Rheinisches Museum für Philologie

RHPhilos. Revue d'Histoire et de Philosophie Religieuses

RHRel. Annales du Musée Guimet. Revue de l'Histoire des Religions

RIA. Rivista dell'Istituto Nazionale d'Archeologia e Storia dell'Arte

RivFil. Rivista di Filologia e di Istruzione Classica 
RivItNum. Rivista Italiana di Numismatica RivScPr. Rivista di Scienze Preistoriche RivStCl. Rivista di Studi Classici RivStudLig. Rivista di Studi Liguri

RLouvre Revue du Louvre et des Musées de France

RM. Mitteilungen des Deutschen Archäologischen Instituts. Römische Abteilung

Rodenwaldt, KdA.4 G. Rodenwaldt, Die Kunst der Antike, 4. Auflage

Roscher, ML. W. H. Roscher, Ausführliches Lexikon der griechischen und römischen Mythologie RQu. Römische Quartalschrift für christliche Altertumskunde und für Kirchengeschichte

RScRel. Recherches de Science Religieuse

RStOr. Rivista di Studi Orientali

SBBerlin Sitzungsberichte der Akademie der Wissenschaften zu Berlin. Phil.-hist. Klasse

SBHeidelberg Sitzungsberichte der Heidelberger Akademie der Wissenschaften. Phil.-hist. Klasse

SBLeipzig Berichte über die Verhandlungen der Sächsischen Akademie der Wissenschaften zu Leipzig. Phil.-hist. Klasse

SBMünchen Sitzungsberichte der Akademie der Wissenschaften zu München. Phil.-hist. Klasse SBWien Sitzungsberichte der Akademie der Wissenschaften in Wien. Phil.-hist. Klasse

SborníkBrno Sborník Prací Filosofické Fakulty Brněnské University

SchwMbll. Schweizer Münzblätter

SNG. Sylloge Nummorum Graecorum

SOsl. Symbolae Osloenses

SovArch. Sovetskaja Archeologija

StCIstor. Studii și Cercetări de Istoria Veche StClas. Studii clasice

StCNum. Studii și Cercetări de Numismaticá

StComApulum Studii și Comunicări. Acta Musei regionalis Apulensis

StDoc. Studia et Documenta Historiae et Juris
SteMat. Studi e Materiali di Storia delle Religioni StEtr. Studi Etruschi

StIt. Studi Italiani di Filologia Classica

StRom. Studi Romani

Thieme-Becker Allgemeines Lexikon der bildenden Künstler. Hrsg. von U. Thieme und F. Becker

ThLZ. Theologische Literaturzeitung

TrAPhAss. Transactions and Proceedings of the American Philological Association

TrCumb. Transactions of the Cumberland and Westmorland Antiquarian and Archaeological Society

TrZ. Trierer Zeitschrift

VDI. Vestnik Drevnej Istorii

VjbllTrier Landeskundliche Vierteljahresblätter der Trierer Gesellschaft für nützliche Forschungen

WaG. Die Welt als Geschichte

Winter, KiB. Kunstgeschichte in Bildern. Neue Bearbeitung. I. Das Altertum

WSt. Wiener Studien

WZMorg. Wiener Zeitschrift für die Kunde des Morgenlandes

ZÄSpr. Zeitschrift für Ågyptische Sprache und Altertumskunde

ZAssyr. Zeitschrift für Assyriologie und Vorderasiatische Archäologie

ZatW. Zeitschrift für alttestamentliche Wissenschaft

ZDMG. Zeitschrift der Deutschen Morgenländischen Gesellschaft

ZDPV. Zeitschrift des Deutschen PalästinaVereins

ZSchwArch. Zeitschrift für schweizerische Archaeologie und Kunstgeschichte

Sonstige Abkürzungen: Bl. Blatt $\begin{gathered}\text { Bll. Blätter } \\ \text { Jb. Jahrbuch, Jahrbücher }\end{gathered} \quad \begin{gathered}\text { Boll. Bollettino } \\ \text { Jber. Jahresbericht }\end{gathered} \begin{gathered}\text { Bull. Bulletin } \\ \text { Rdsch. Rundschau }\end{gathered} \quad$ J. Journal

Rev. Review, Revue Riv. Rivista 
